\title{
HAND GESTURE RECOGNITION SYSTEM FOR HUMAN COMPUTER INTERACTION USING CONTOUR ANALYSIS
}

\author{
Ann Abraham Babu ${ }^{1}$, Satishkumar Varma ${ }^{2}$, Rupali Nikhare ${ }^{3}$ \\ ${ }^{I}$ ME student, Department of Information Technology, PIIT, Maharashtra, India \\ ${ }^{2}$ Associate Professor, Department of Information Technology, PIIT, Maharashtra, India \\ ${ }^{3}$ Assistant Professor, Department of Computer Engineering, PIIT, Maharashtra, India
}

\begin{abstract}
Hand gesture recognition is a very challenging topic for real life applications because of its requirements on the robustness, accuracy and efficiency. This paper describes a system that enable a user to perform computer operations using hand gesture with a simple web camera as input device. This system involves four phases namely image acquisition, image pre-processing, feature extraction and gesture recognition. In the first phase, the input image is acquired with the help of a camera. In the second phase, the skin color of hand region is detected using HSV color space and morphological operations such as erosion and dilation are performed to remove noise followed by smoothing and thresholding of hand image. In Feature extraction phase, contours of hand image are detected. Finally, Gesture recognition phase includes recognizing hand gestures using contour analysis by comparing Auto-Correlation Function (ACF) amongst the contours and if they are close, then calculate Inter-Correlation Function (ICF) to truly determine similarity. Each recognized gesture is assigned with the corresponding action.
\end{abstract}

Keywords: Hand gesture recognition, human computer interaction, contour analysis, HSV color space.

\section{INTRODUCTION}

Gestures are bodily actions made by humans to convey meaningful information to others. It comes easily to human and so using these gestures as a mode of interaction will help humans to interact with computer easily. A gesture is scientifically categorized into two distinctive categories: dynamic and static [8]. A dynamic gesture is intended to change over a period of time whereas a static gesture is observed at the spurt of time. A waving hand means goodbye is an example of dynamic gesture and the stop sign is an example of static gesture. In this paper we mainly focus on hand gestures. The primary goal of hand gesture recognition system is to create a system which can identify specific hand gestures and use them to convey information or device control.

The remaining part of this paper is organized as follows. Section 2 describes existing approaches for recognizing hand gesture. System overview is presented in Section 3. Section 4 explains the algorithm used for hand gesture recognition process. Section 5 describes the proposed system. Experimental setup and Experimental results are presented in section 6 and section 7 respectively. Section 8 gives the conclusions.

\section{RELATED WORK}

Many works have been done on hand gesture recognition using different techniques. This section gives a quick review on some of the previous work done. Meenakshi Panwar and Pawan Singh Mehra [1] proposed a novel approach of hand gesture recognition based on detection of some shape based features. First step includes pre-processing and segmentation of hand image to reduce the noise from captured input image. In the second step it detects the orientation of hand shapes. In the Third step it calculates the essential features required for generating the bit sequence for different hand shapes. And finally recognition and interpretation of different hand gestures. The weakness of this method is that it does not follow any systematic approach to define certain parameters for gesture recognition and also requires hand to be placed straight horizontal or straight vertical to be recognized accurately. Meenakshi Panwar [2] proposed approach that uses some pre-processing steps for removal of background noise and employs K-means clustering for segmentation of hand object so that only segmented hand objects or cluster is to be processed in terms of shape based features. It calculates some essential features required for hand pattern detection and for generating the 7 bit sequence for 36 different hand shapes. The weakness of this method is that it does not follow any systematic approach for gesture recognition. Similarly Meenakshi Panwar [3] proposed another approach that uses k-mean clustering for segmenting the hand object and identifies around 45 different gestures on the bases of 5 bit binary string. Ashis Pradhana, M.K.Ghosea and Mohan Pradhana [4] presented a very simple and efficient approach for recognizing the hand gesture that represents numbers from zero to nine. The design of above discussed hand gesture recognition system is broadly divided into two phase namely, preprocessing phase and classification phase. The weakness of this approach is that the designed system is able to classify only the static images. S. Nagarajan, Dr. T. S. Subashini, Dr. V. Ramalingam [5], presents a real time finger counter for counting the number of fingers (1-5) shown in a hand gesture. In the first phase, the gesture video is captured 
using a camera in real time. The skin color of hand region is detected using HSV color space and morphological operations are performed in the third phase. In the feature extraction phase, the convex hull method is used to detect the boundary points of the segmented binary hand image. Finally, the recognition phase recognizes the gesture number based on the extracted features. The main drawbacks of this proposed work are hand pose orientation. The method described in paper [9] uses compactness value to recognize hand gestures. The weakness of this method is that if two hand shapes with same compactness value exists then it classifies these two different shapes as same.

\section{SYSTEM OVERVIEW}

The architecture diagram of hand gesture recognition system is shown in Figure 1 which consists of four phases namely, Image Acquisition phase, Image Pre-processing phase, Feature Extraction phase and Gesture Recognition phase.

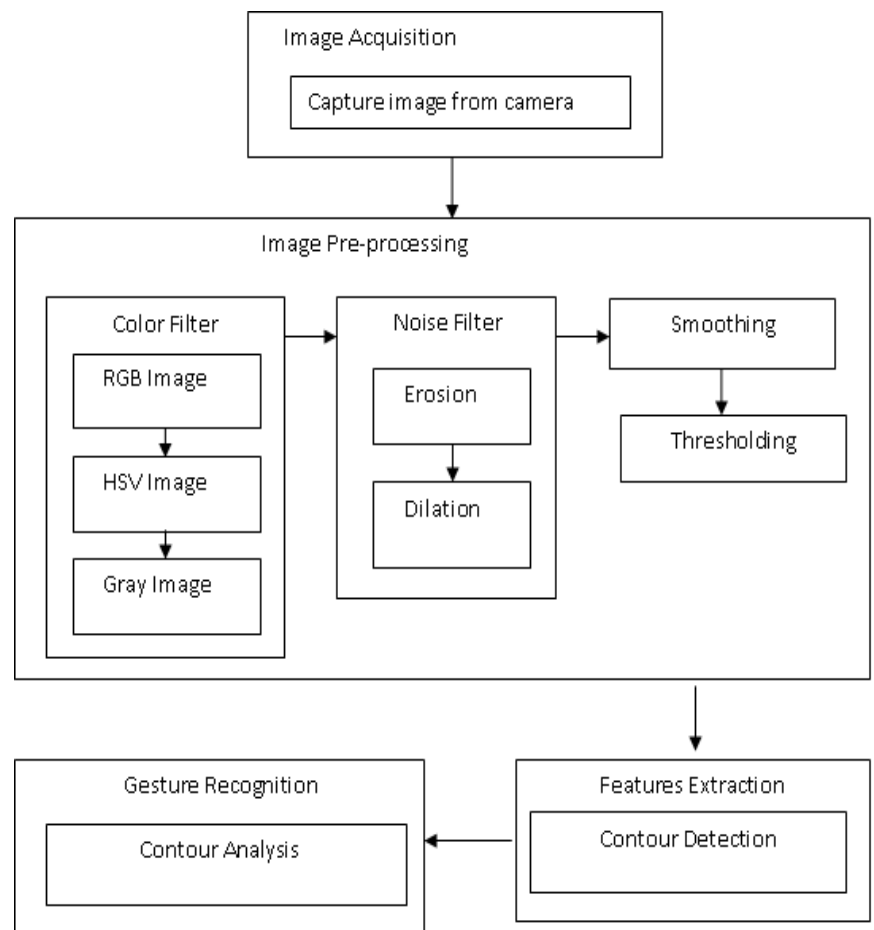

Fig 1: Architecture diagram of Hand Gesture Recognition System

\subsection{Image Acquisition}

Image Acquisition is accomplished by means of a camera, which captures images frame by frame.

\subsection{Image Pre-processing}

The main function of this phase is to extract the hand image from its background. It involves color filter, noise filter, smoothing and thresholding.

\subsection{Feature Extraction}

This phase finds and extracts features of hand image. Here hand contour is used as a feature because contour contains the necessary information on the object shape.

\subsection{Gesture Recognition}

Gesture Recognition phase includes recognizing hand gestures with the help of extracted features.

\section{METHODOLOGY}

The Flowchart of implemented algorithm is shown in Figure 2.

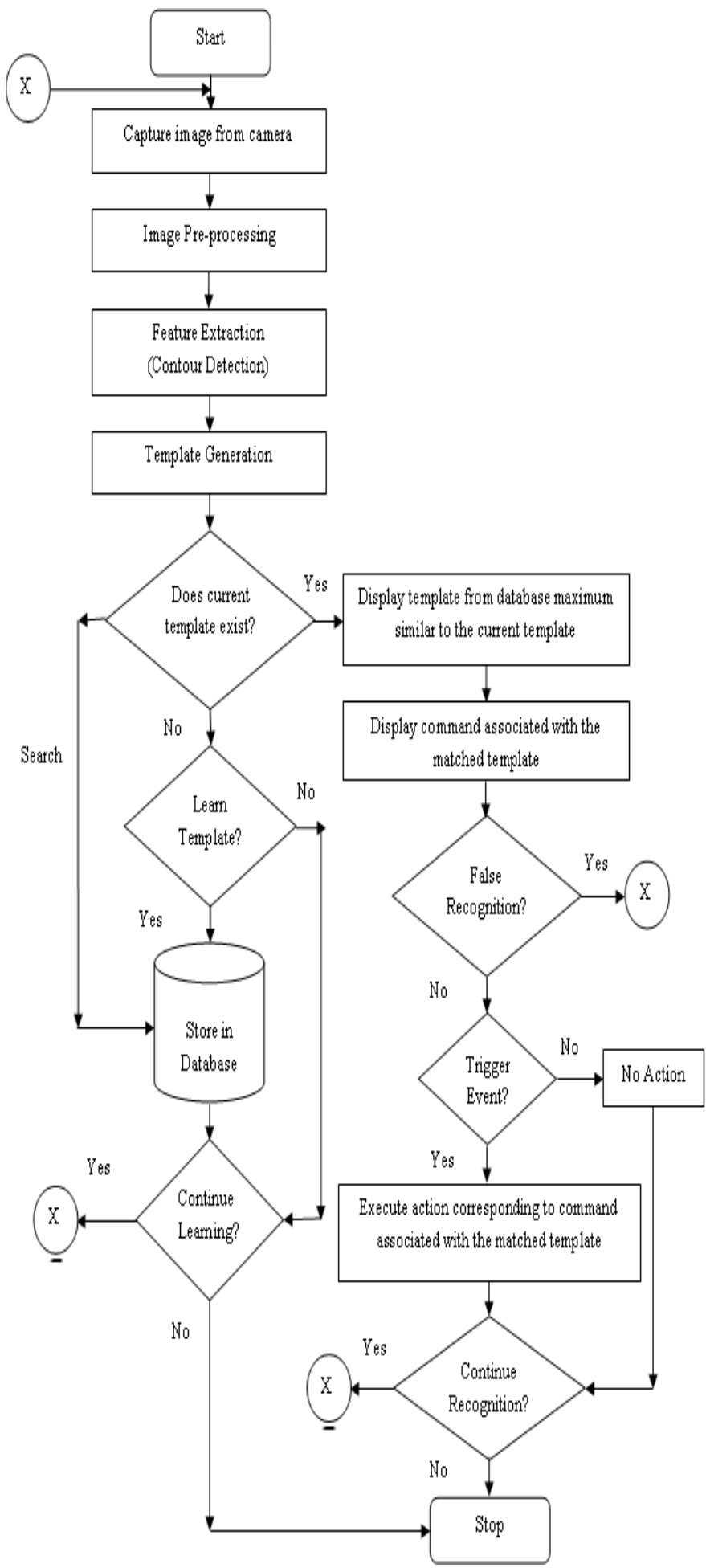

Fig 2: Flowchart of the implemented algorithm. 
The algorithm for hand gesture recognition system is given below, where the input will be hand gesture and the output will be execution of action assigned to a particular hand gesture.

\section{Input: Hand Gesture}

Output: Assigned Action Executed

Step 1: Capture the input image from camera.

Step 2: Convert input RGB image into HSV image.

Step 3: Convert HSV image into gray image.

Step 4: Perform morphological operations such as erosion and dilation to reduce noise followed by smoothing and thresholding of image.

Step 5: Extract contours of hand image.

Step 6: Store contours as contour templates.

Step 7: Gesture is recognized using contour analysis by comparing ACF and ICF values.

Step 8: For each recognized gesture, different events are linked to perform various events by the computer.

\section{MODULE DESCRIPTION OF PROPOSED}

\section{SYSTEM}

The different modules of project are discussed in this section

\subsection{Web cam Access Module}

It consists of program to access web camera in order to take input from the web cam. iBall CHD 20.0 webcam is used for capturing hand image. Image is captured in RGB colorspace format. By clicking on start button in GUI, the input image is captured by web cam. Output of this shows "Camera Started". By clicking on stop button in GUI, user can stop the web cam.

\subsection{Image Pre-processing Module}

It takes the image from the web cam access module and processes it. In this step, the pixels of user's hand are extracted from the input image and converted into HSV image and then into gray image. To remove noise, morphological operations such as erosion and dilation and smoothing are performed. Firstly image erosion is applied, which trims down the image area where the hand is not present. The second stage is to apply image dilation which effectively enlarges the area image pixels that have not been eroded. The functions in OpenCV for erode and dilate are cvErode() and cvDilate() respectively. cvSmooth() function is used for smoothing. The Gaussian smooth step is used to remove noise in the image and leave only the main contours. In Thresholding, we make a final decision about the pixels in an image by rejecting those pixels below or above some value while keeping the others. The OpenCV function cvThreshold() is used for thresholding. This step outputs binary image, in which only the pixels belonging to hand have value 1 and the other have value 0 . By clicking on start process button in GUI, the hand gesture processing starts. By clicking on stop button in GUI, user can stop the processing. Figure 3 shows hand image obtained at preprocessing phase.

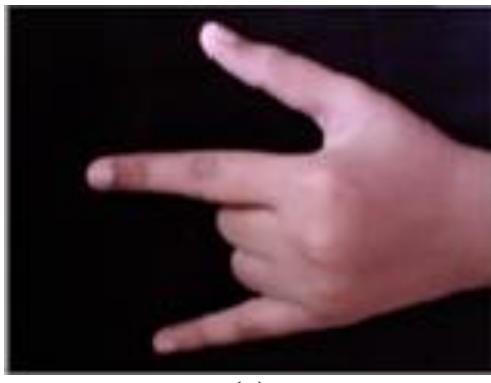

(a)

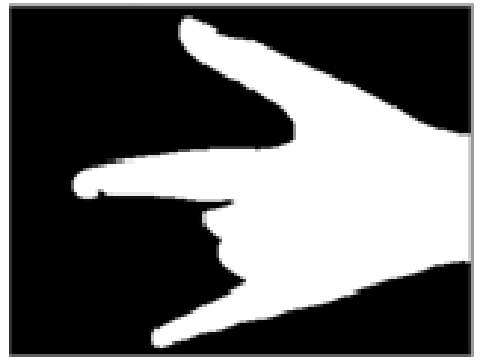

(b)

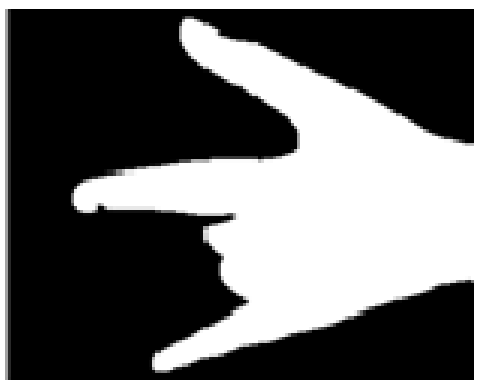

(c)

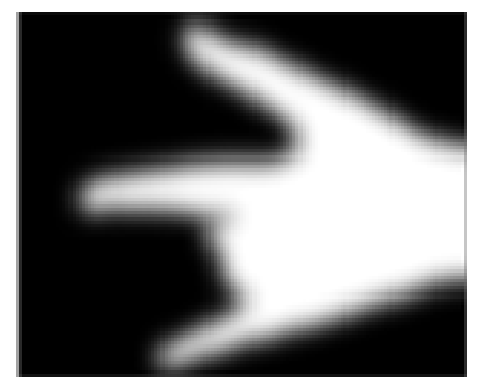

(d)

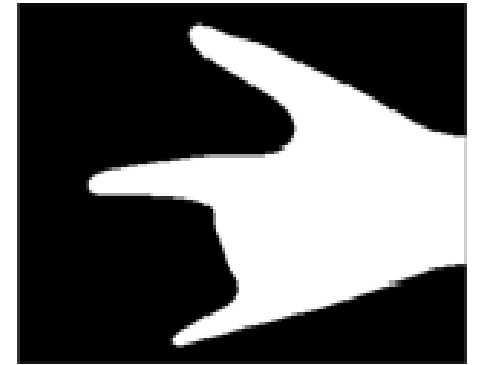

(e)

Fig 3 (a): Original Image, (b): Gray Image, (c): Image after morphological operations, (d): Smooth Image, (e): Threshold Image. 


\subsection{Feature Extraction Module}

This module extracts feature from hand image for gesture recognition and extracted features will be feed as an input for recognition process. The shape of the contour is an important property that can be used to distinguish hand gestures. Contour of hand image are extracted as feature. These contours are stored as contour templates which will be used for gesture recognition

\subsection{Template Loading, Learning and Saving}

\section{Module}

This module stores contour template in bin file and each of these templates exists only as a vector of points with an $\mathrm{x}$ and $y$ coordinate. The database file is called "Templates.bin". It loads previously stored templates from database and also allows the system to learn new templates. In learn template module, user can learn new template by clicking on learn template button in GUI. The contours for given real time images are detected first and particular pattern as a template is stored in database. The database consists of 40 stored templates.

\subsection{Hand Gesture Recognition module}

It contains code for hand gesture recognition. After completion of learning phase, the next that follows is recognition phase. The hand gesture is recognized using contour analysis [7]. During recognition phase when an hand gesture is given as input to the system to be recognized, contour for given hand gesture are detected first and then it searches in database for contour template similar to the given contour by comparing ACF amongst the contours and if they are close, then calculates ICF to truly determine similarity and finally returns template maximum similar to the given contour [7]. When a given pattern is matched with the contour template, assigned command is displayed for that contour template.

\subsection{Event Handling Module}

On selecting the event trigger option in GUI, action assigned to command corresponding to a particular contour template is executed. In our project, action assigned includes Open power-point, F5 key press, Right Arrow key press, Left Arrow key press, Open Ms-word, Open Ms-Excel, Open Google chrome and Close application.

\section{EXPERIMENTAL SETUP}

The user interface is designed using the image processing techniques implemented in $\mathrm{C \#}$ with the use of OpenCV Library. The experimental setup requires a web camera to capture the gesture formed by the user preferably with a plain black background. It consists of a stand to place camera so that hand gesture can be captured without any disturbance. The system is developed using a generic tool for Computer Vision applications, EmguCV package in OpenCV [6].

\section{EXPERIMENTAL ANALYSIS}

We performed the experiment for eight kind of gesture which are shown in Figure 4.

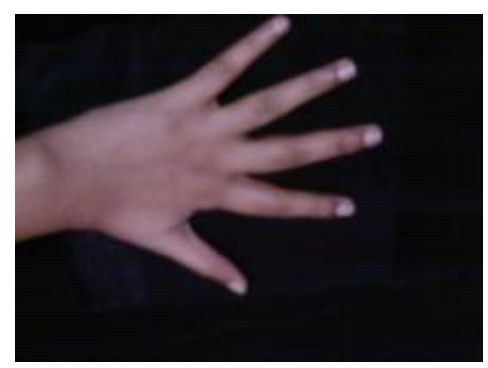

(a) Ms-powerpoint

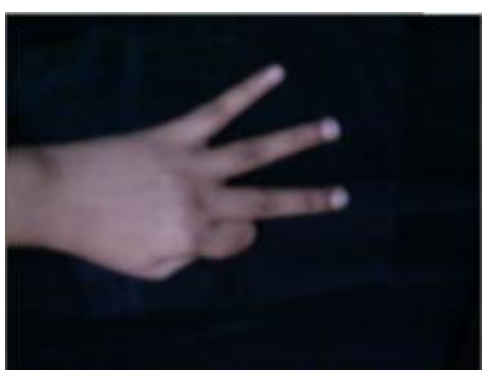

(b) F5 Keypress

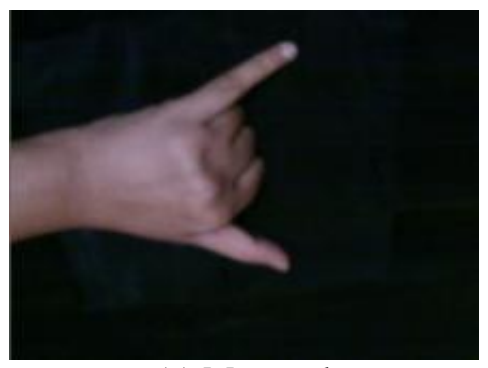

(c) Ms-excel

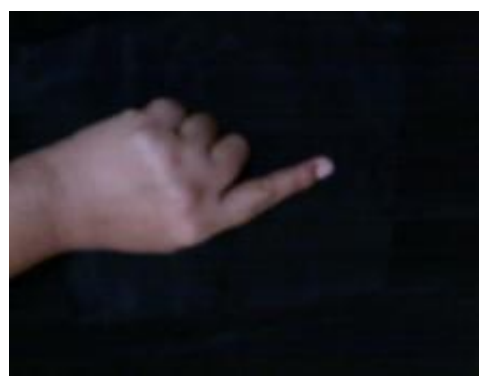

(d) Right Arrow

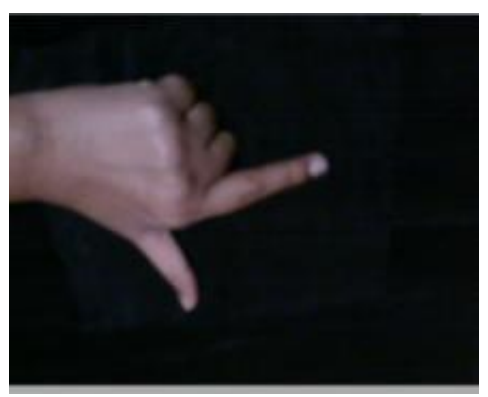

(e) Left Arrow 


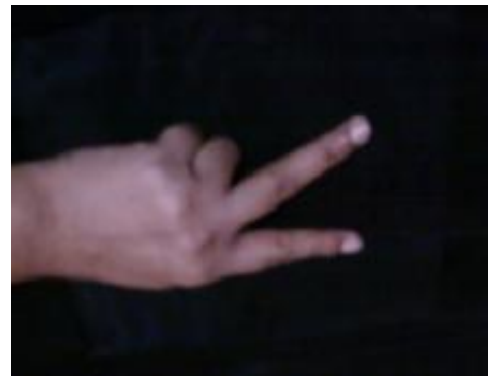

(f) Google Chrome

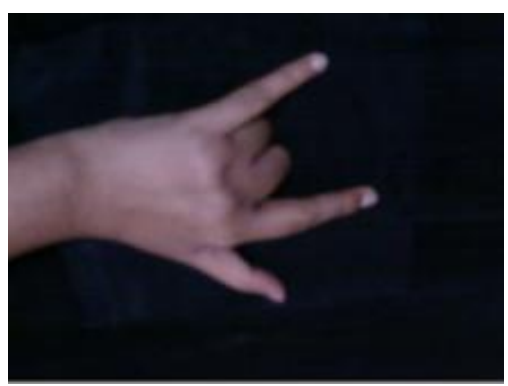

(g) Ms-word

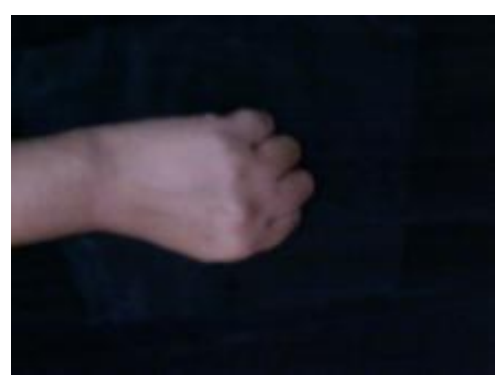

(h) Close

Figure 5 shows the face of proposed system along with hand gestures and their corresponding Human Computer Interactions. The experimental results are tabulated in Table 1.

Table 1: Hand Gesture Recognition Rate

\begin{tabular}{|c|c|c|c|c|}
\hline $\begin{array}{l}\text { Gesture } \\
\text { Events }\end{array}$ & $\begin{array}{l}\text { No. of } \\
\text { Input } \\
\text { samples }\end{array}$ & $\begin{array}{l}\text { No. of } \\
\text { recognize } \\
\text { d samples }\end{array}$ & \begin{tabular}{ll}
\multicolumn{2}{l}{ Recogniti } \\
on Rate \\
$(\%)$
\end{tabular} & $\begin{array}{l}\text { Average } \\
\text { Recogniti } \\
\text { on Rate } \\
(\%)\end{array}$ \\
\hline $\begin{array}{l}\text { Open } \\
\text { power- } \\
\text { point }\end{array}$ & 10 & 09 & $90 \%$ & \multirow{8}{*}{$95 \%$} \\
\hline $\begin{array}{ll}\text { F5 } & \text { Key } \\
\text { press } & \end{array}$ & 10 & 09 & $90 \%$ & \\
\hline $\begin{array}{l}\text { Open Ms- } \\
\text { excel }\end{array}$ & 10 & 10 & $100 \%$ & \\
\hline $\begin{array}{l}\text { Right } \\
\text { Arrow }\end{array}$ & 10 & 09 & $90 \%$ & \\
\hline Left Arrow & 10 & 09 & $90 \%$ & \\
\hline $\begin{array}{l}\text { Open Ms- } \\
\text { word }\end{array}$ & 10 & 10 & $100 \%$ & \\
\hline $\begin{array}{l}\text { Open } \\
\text { Google } \\
\text { Chrome }\end{array}$ & 10 & 10 & $100 \%$ & \\
\hline $\begin{array}{l}\text { Close } \\
\text { application }\end{array}$ & 10 & 10 & $100 \%$ & \\
\hline
\end{tabular}

Fig 4: Hand Gesture Vocabulary

The performance evaluation result is shown in Chart 1 .

Recognition Rate of Gesture Events

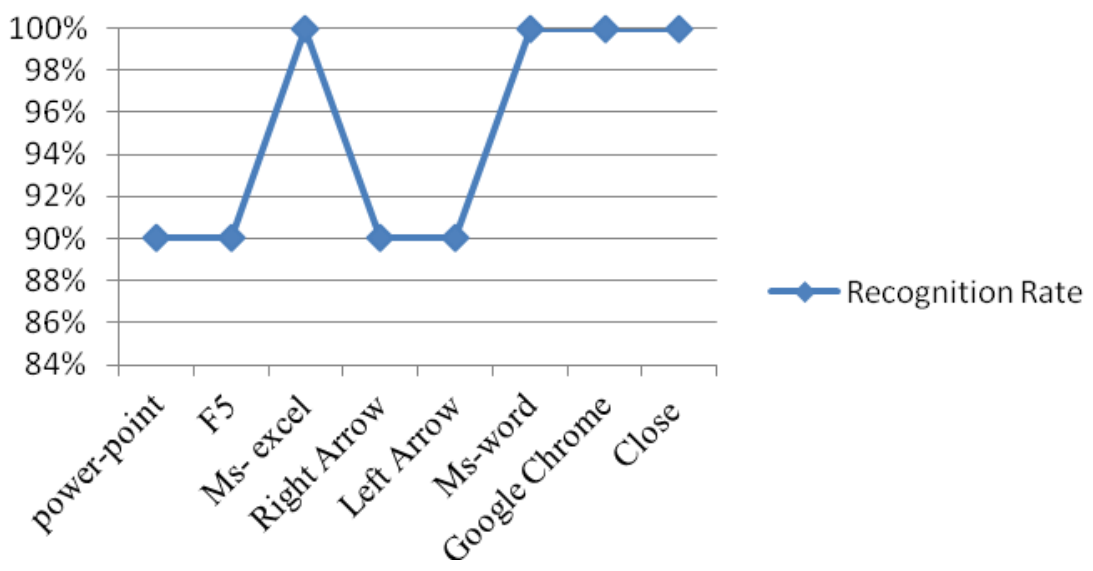

Chart 1: System Performance and Evaluation Results 


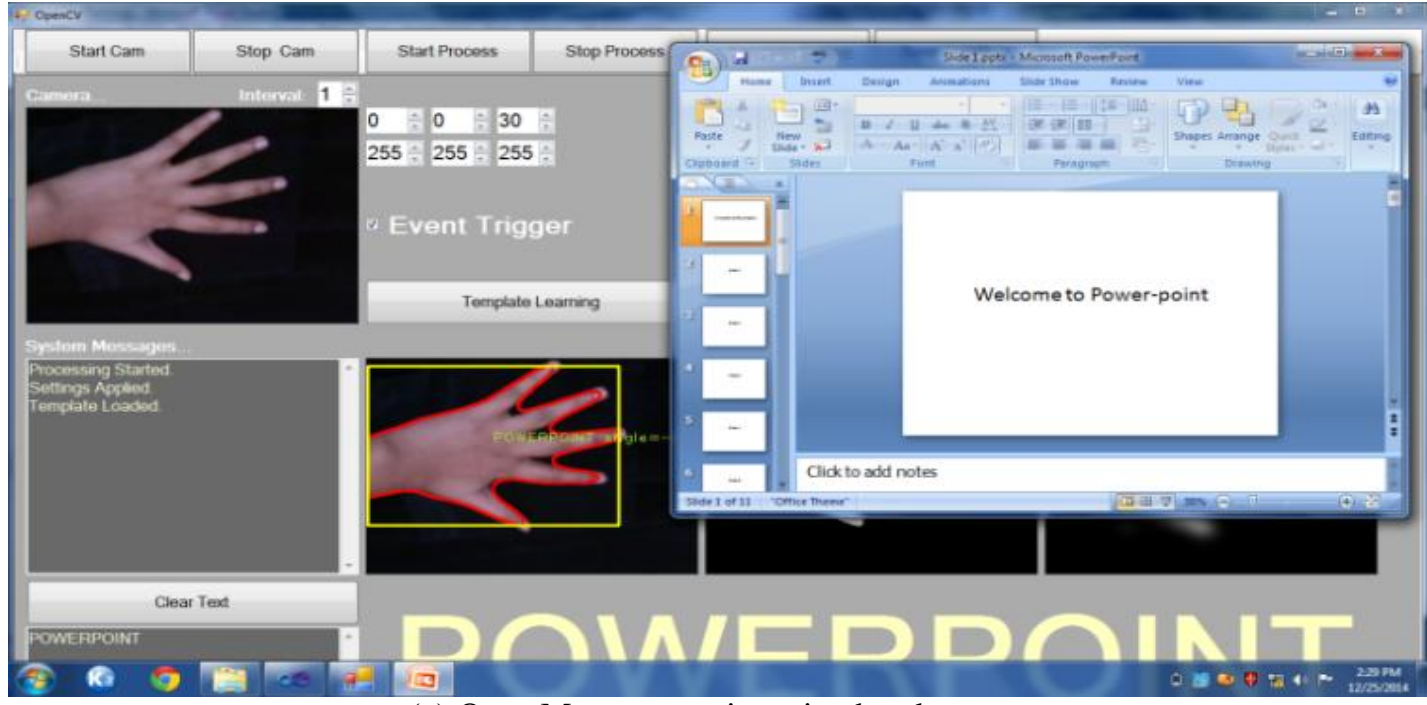

(a) Open Ms-power point using hand gesture

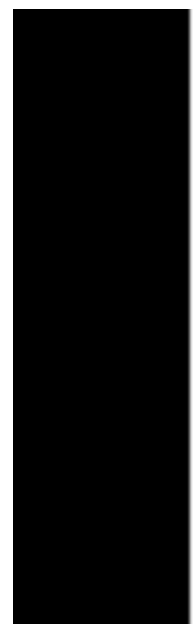

\section{Welcome to Power-point}

(b) F5 Key press in power point using hand gesture

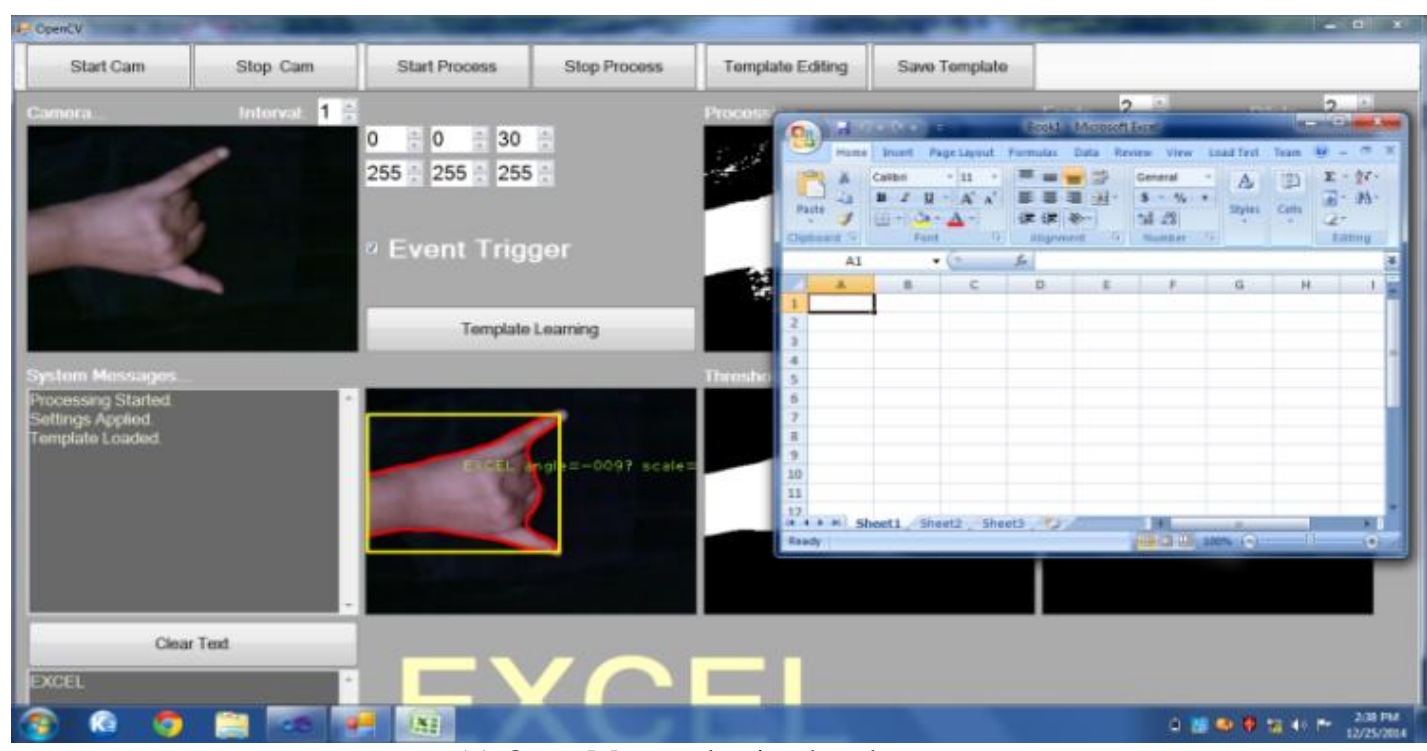

(c) Open Ms-excel using hand gesture 


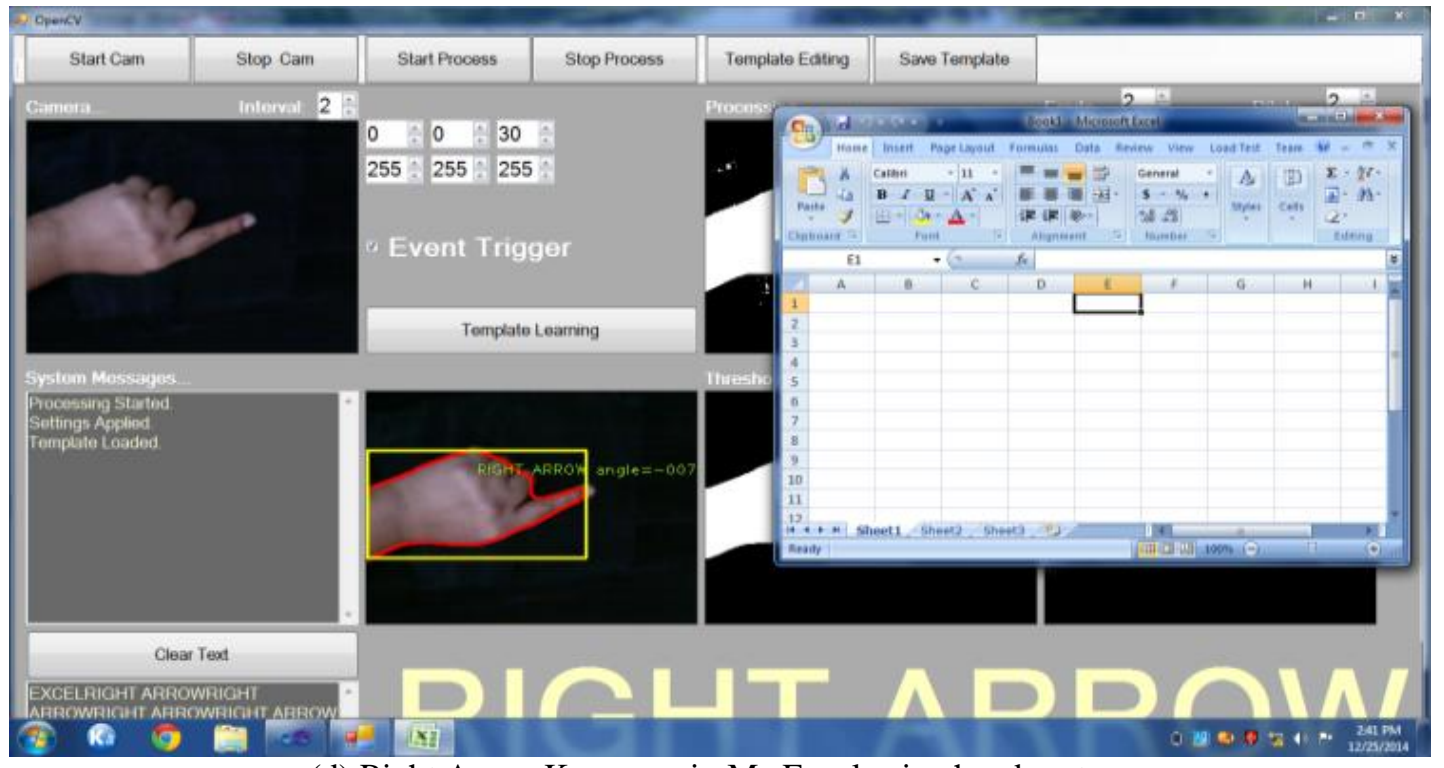

(d) Right Arrow Key press in Ms-Excel using hand gesture

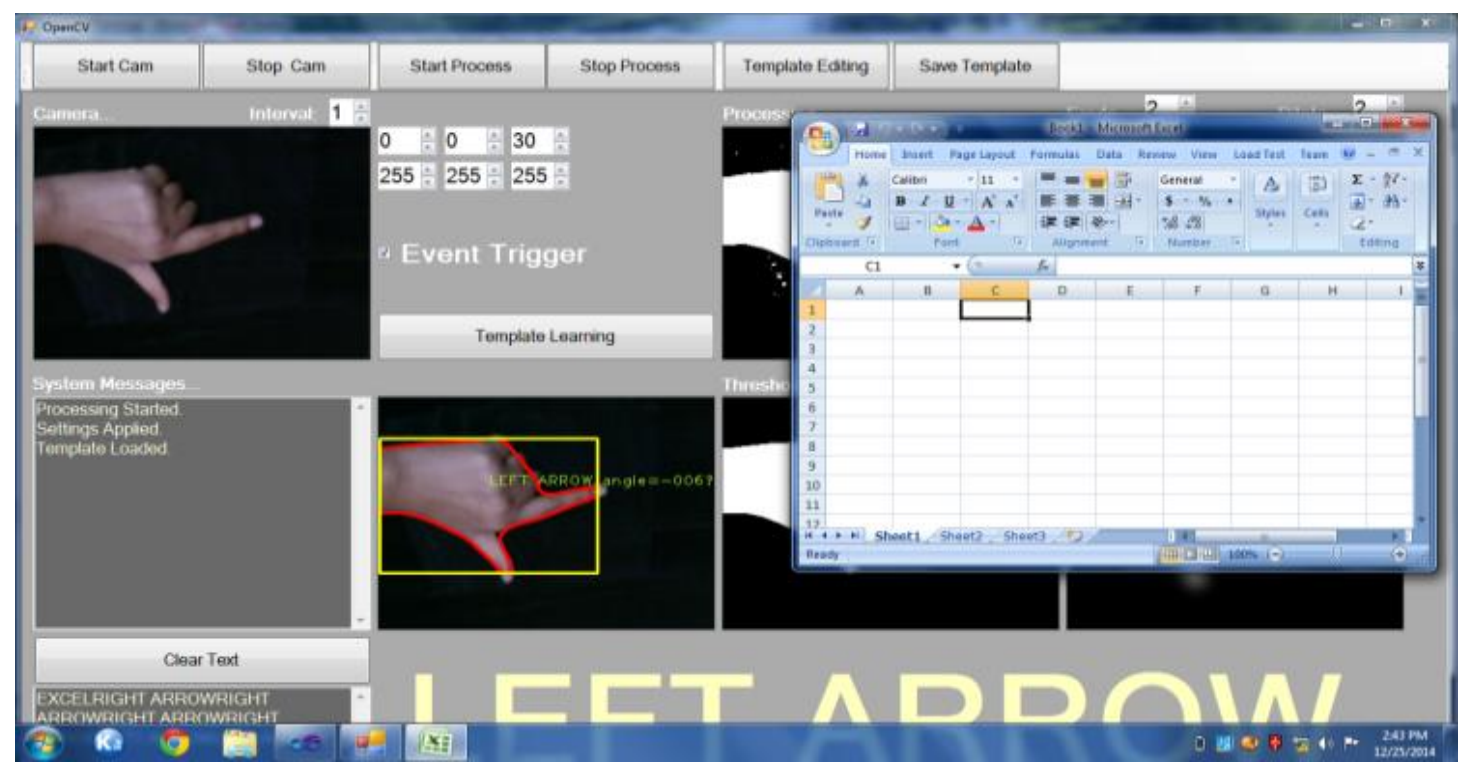

(e) Left Arrow Key press in Ms-excel using hand gesture

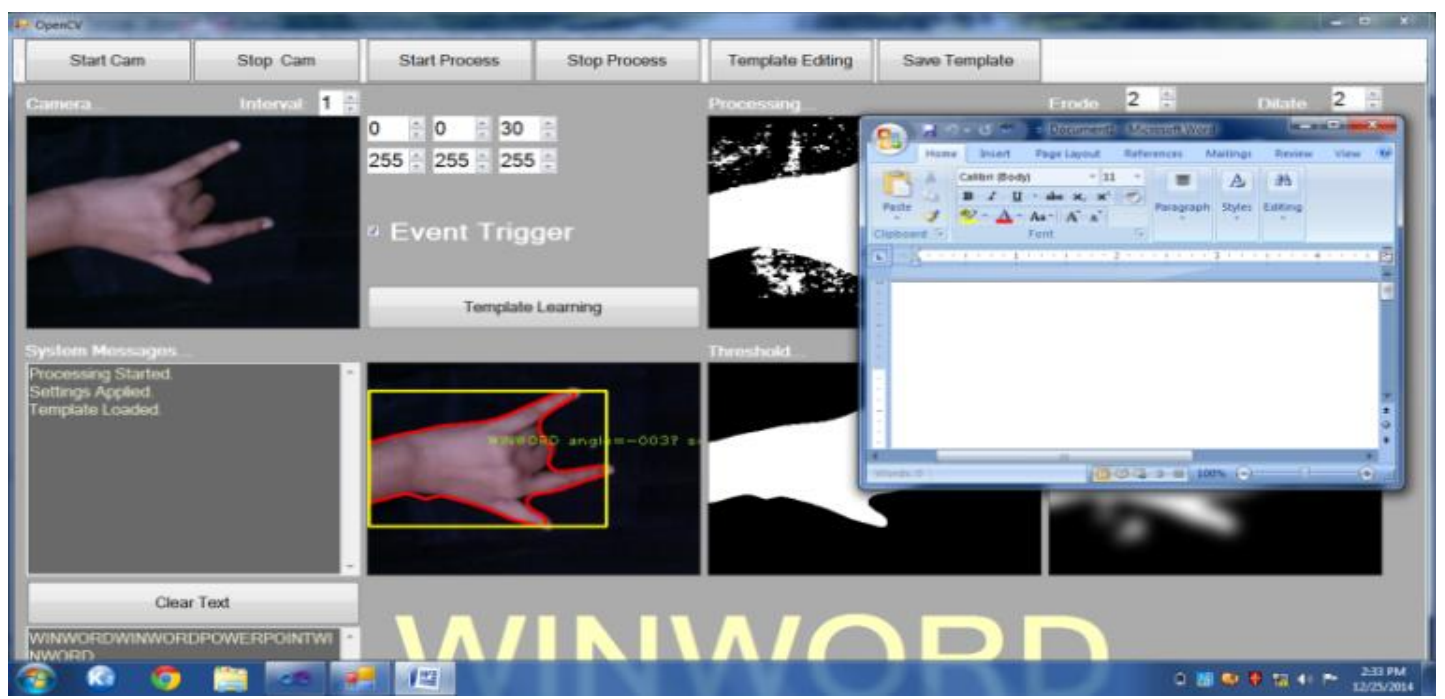

(f) Open Ms-word using hand gesture 


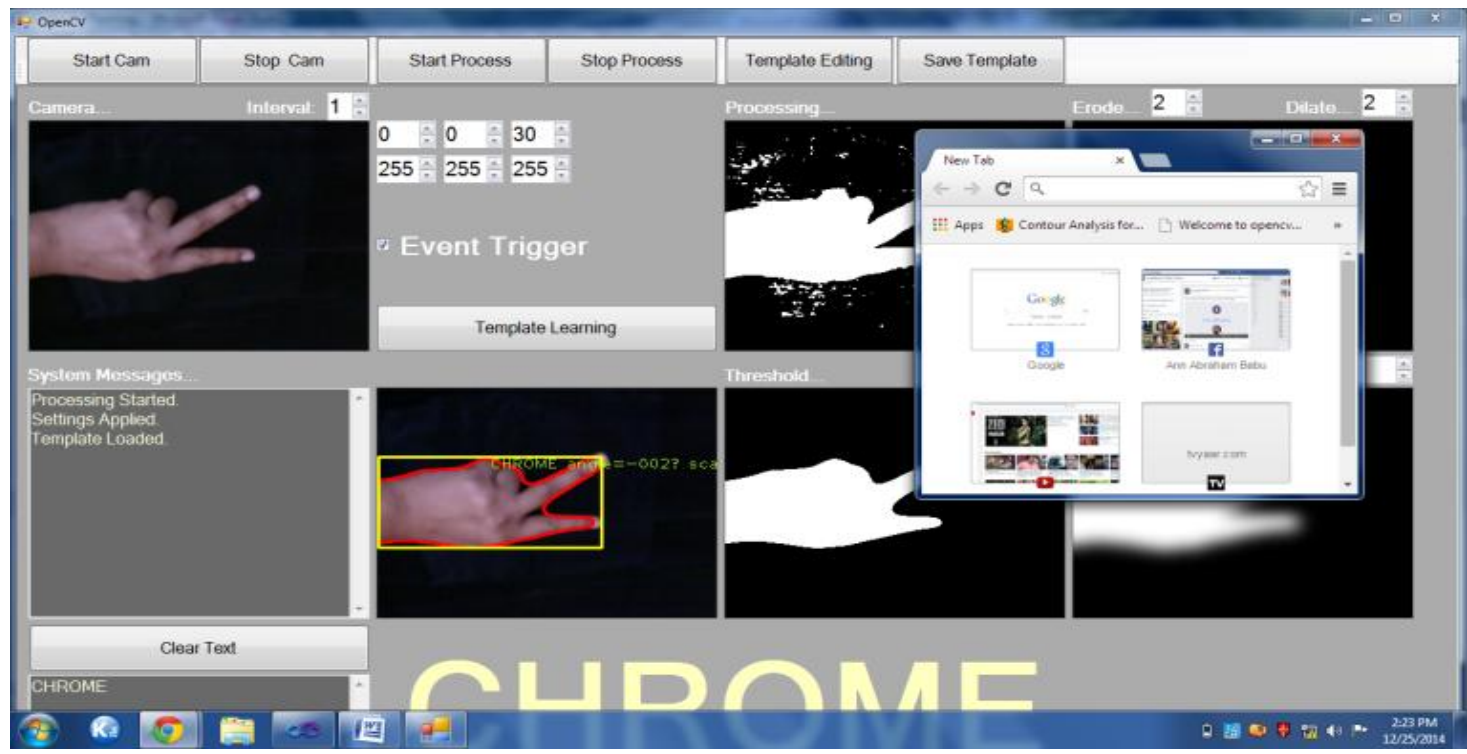

(g) Open Google chrome using hand gesture

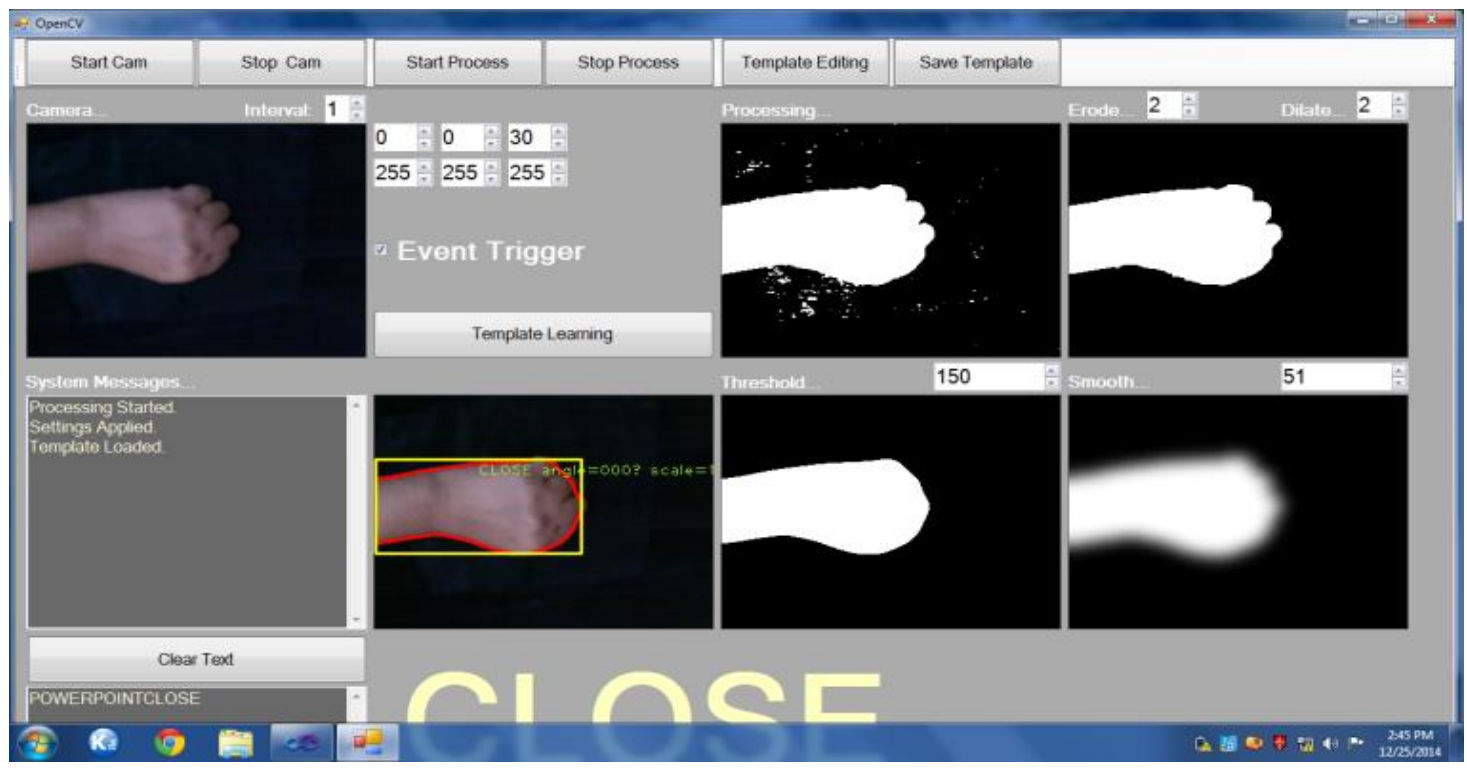

(h) Close Application using hand gesture

Fig 5: Hand Gestures and their corresponding HCI

\section{CONCLUSION}

This paper describes a system that controls computer applications with the help of hand gestures. The proposed approach offers a unique method to identify hand gesture using contour analysis. Experimental results show that the average recognition rate is $95 \%$.

The current system gives best result in a plain black background. Thus the current system puts lots of constraints on the user for successful working. The future work includes reducing these constraints so that the system is usable in more scenarios. Further enhancement of the technique proposed is possible using both hands for performing different computer operations. Experiments need to be done on a larger scale so that results can be more accurate.

\section{ACKNOWLEDGEMENTS}

I thank the Lord Almighty for his grace and blessings which helped me to complete this study. I remain immensely obliged to my guide Dr. Satishkumar Varma and Professor Rupali Nikhare for their guidance and suggestions during the preparation of this technical paper. I thank my beloved parents, Mr. Babu Varghese and Mrs. Saramma Babu Varghese for their cooperation and support throughout. Finally I would like to thank all who contributed and helped me in this project.

\section{REFERENCES}

[1]. Meenakshi Panwar and Pawan Singh Mehra, "Hand Gesture Recognition for Human Computer Interaction", IEEE International Conference on Image Information Processing (ICIIP 2011), Waknaghat, India, Nov 2011. 
[2]. Meenakshi Panwar, "Hand Gesture based Interface for Aiding visually Impaired", IEEE International Conference on Image Information Processing (ICIIP 2012), Noida, India.

[3]. Meenakshi Panwar, "Hand Gesture Recognition based on Shape Parameters", International Conference on Computing, Communication and Applications (ICCCA), 2012.

[4]. Ashis Pradhana, M.K.Ghosea and Mohan Pradhana "A Hand Gesture Recognition using Feature Extraction" International Journal of Current Engineering and Technology ISSN 2277 - 410613 -Nov. 2012.

[5]. S. Nagarajan, Dr. T. S. Subashini, Dr. V. Ramalingam, "Vision Based Real Time Finger Counter for Hand Gesture Recognition", CPMR-IJT: International Journal of Technology Vol. 2, No. 2, December 2012.

[6]. G. Bradski and A. Kaehler, "Learning OpenCV Computer Vision with the OpenCV Library", O'Reilly Publications, 2008.

[7]. Namrata.S.Mandvikar and Sunita Jadhav, "Augmented Reality Learning System with Contour Analysis" IJIIT, ISSN:2278 9057.

[8]. M.R. Ramjan, R.M. Sandip, P.S. Uttam, W.S. Srimant, "Dynamic Hand Gesture Recognition and Detection for Real Time Using Human Computer Interaction" IJARCSMS, ISSN: 2321-7782.

[9]. Amornched Jinda-apiraksa, Warong Pongstiensak, and Toshiaki Kondo, "A Simple Shape-Based Approach to Hand Gesture Recognition", in Proceedings of IEEE International Conference on ECTI-CON, Thailand, pages 851-855, May 2010.

\section{BIOGRAPHIES}

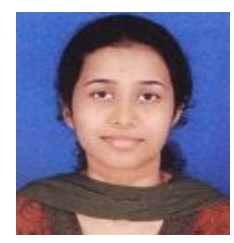

Ann Abraham Babu is pursuing M.E. in Information Technology from PIIT. She obtained B. E. in Computer Science from PIIT in the year 2011. She has published papers in International Journal. Her research interest is in Image Processing and Human Computer Interaction.

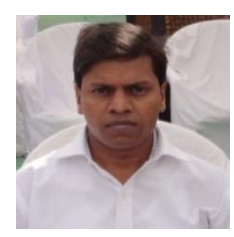

Satishkumar L. Varma has received his B.Tech and M. Tech. degrees in June 2000 and January 2004 respectively in Computer Engineering from DBATU, Lonere, India. He has received $\mathrm{Ph}$. D degree in Computer Science and Engineering from SRTMU, Nanded, India. He has worked for 2 years as Lecturer in the Department of Computer Engineering, RMCET, Devrukh, around 3 years as a Lecturer in the Department of Computer Engineering, SAKEC Mumbai and 5 years as Assistant Professor and Head of Department of Information Technology, DBIT, Mumbai. Currently he is Associate Professor and Head of Department of Information Technology, PIIT, Navi Mumbai, India. He has published one book chapter and around 20 papers in referred National as well as International Conferences and Journals including IEEE, Springer LNCS, IET, IJACSA and IJIP. His research interest includes Multimedia Systems, Digital Image Processing and Computer Vision.

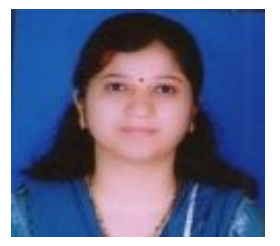

Rupali Nikhare is working as an Assistant Professor in Computer Engineering Department at PIIT New Panvel. She obtained M. E. in Computer Engineering and B. E. in EXTC. She has ten years of teaching experience. Her area of interest is in DSP, IP, CV, Robotics, E-commerce. 